\title{
Effects of Plumbagin on Proliferation, Invasion and Migration of Glioma Cell Line U-118
}

\author{
CHUN YAN GAO*, ZHEN GUI NIE, SHU WANG AND SHI QUAN WANG ${ }^{1}$ \\ Department of Laboratory Science, ${ }^{1}$ Department of Clinical Medicine, Yanjing Medical College, Capital Medical University, \\ No. 4, Dadong Road, Shunyi District, Beijing 101300, China
}

\section{Gao et al.: Plumbagin Effects on Glioma Cell Line U-118}

\begin{abstract}
To observe the inhibitory effect of plumbagin on the invasion and migration of glioma cells U-118 and to explore the possible mechanism of its action. U-118 cells were cultured in medium with different concentrations of plumbagin and the cells were counted and morphological changes were observed under the microscope after $24 \mathrm{~h}$ of incubation and the cell proliferation was detected by 3-(4,5-dimethylthiazol2-yl)-2,5-diphenyl tetrazolium bromide, the effect of plumbagin on cell migration was detected by cell scratching assay and the effect of cell invasion was detected by Transwell method. Western blotting was used to detect the expression of matrix metalloproteinase-2, matrix metalloproteinase-9 and p21 protein. The results showed that the morphology of $U-118$ cells could be changed by plumbagin and it was dependent on the concentration; 3-(4,5-dimethylthiazol-2-yl)-2,5-diphenyl tetrazolium bromide results showed that plumbagin significantly inhibited the growth of U-118 cells and its inhibitory ability was positively correlated with the concentration; scratch assay results showed that the planar motility of cells decreased significantly with the increase of plumbagin concentration; Transwell Western blotting results showed that plumbagin administration could inhibit the expression of matrix metalloproteinase-2 and matrix metalloproteinase-9 proteins in U-118 cells, but significantly increased the expression of P21 and there was a positive correlation with the concentration. Plumbagin can inhibit the proliferation of U-118 cells and suppress cell invasion and the mechanism may be related to the inhibition of matrix metalloproteinase-2, matrix metalloproteinase-9 and p21 expressio.
\end{abstract}

Key words: Plumbagin, glioma, proliferation, invasion, migration

Gliomas, as the most common intracranial malignant tumors, account for about $50 \%$ of primary brain tumors and the mortality of high grade gliomas is high ${ }^{[1,2]}$. The classic classification of glioma can be divided into four grades: grade I and grade II are low grade glioma, while grade III and grade IV are high grade glioma. Among the four grades of glioma, high grade glioma is the most common malignant central nervous system tumor ${ }^{[3,4]}$. The clinical therapeutic effect of glioma is still very poor, which is due to the high proliferation and invasiveness of tumor cells. Although great progress has been made in surgical resection, chemotherapy and radiotherapy, the prognosis of glioma patients is still poor, which is still a threat to human health ${ }^{[5]}$. Therefore, there is an urgent need for effective diagnosis and treatment of glioma treatment strategy. As a kind of $\alpha$ - $(1,4-)$ naphthoquinone compound, plumbagin has a wide range of biological activities. In recent years, it has been found that plumbagin can inhibit the proliferation of breast cancer, lung cancer, osteosarcoma, liver cancer, leukemia, ovarian cancer and other tumor cells, which shows its good anti-tumor prospects and has attracted extensive attention ${ }^{[4-7]}$. Previous studies have shown that plumbagin can inhibit the proliferation of glioma cells and induce the apoptosis process. However, there are few reports about its effect on the invasion and metastasis of glioma cells. The aim of this study was to investigate the inhibitory effect of plumbagin on the invasion and metastasis of glioma cells and to provide experimental data for the application of plumbagin in the treatment of glioma. Human glioma U-118 cell line was purchased from the cell bank of Shanghai Institute of cell biology, Chinese Academy of Sciences. The cells were cultured a $5 \% \mathrm{CO}_{2}$ incubator at 37\% with Dulbecco's Modified Eagle Medium (DMEM) high glucose medium and $10 \%$ Fetal bovine serum (FBS) (1\% l-glutamine and $1 \%$ penicillin streptomycin). The cells in logarithmic growth phase were used in the experiment. Reagents and instruments: plumbagin, Dimethyl Sulfoxide (DMSO) and 3-(4,5-dimethylthiazol-2-yl)-2,5-diphenyl 
tetrazolium bromide (MTT) were purchased from sigma company (Sigma, USA); DMEM high glucose medium and trypsin (GIBCO, USA); FBS (Shanghai, biyuntian); chemiluminescence detection kit (Pierce, USA); Matrigel (BD, USA); Transwell chamber (Chongqing Bopei Biotechnology Co., Ltd.); Vascular endothelial growth factor (VEGF), MMP-2, MMP-9 antibody (Abcam company); $\beta$-Catenin antibody (Abcam, USA); polyvinylidene fluoride (PVDF) membrane (Mindray biofilm technology, USA). U-118 cells were cultured in DMEM complete medium containing $10 \%$ FBS in cell incubator at $37 \%$ and $5 \% \mathrm{CO}_{2}$ saturation humidity. Cell monolayer adherent growth, change the culture medium every 2-3 d, when it reaches the bottom of the culture bottle, passaged according to the ratio of $1: 3$. U-118 cells in logarithmic growth phase were cultured and the cell concentration was adjusted to $1.0 \times 10^{7} / 1$. the cells were seeded on 96-well cell culture plate with $100 \mu \mathrm{l}$ per well. After the cells adhered to the plate, the culture medium was discarded. After being treated with different concentrations of plumbagin for $24 \mathrm{~h}, 20 \mu \mathrm{l}$ of prepared MTT solution was added into each well, and incubated at $37 \%$ for $4 \mathrm{~h}$. The supernatant was discarded. $150 \mu 1$ DMSO was added into each well and the oscillation was $10 \mathrm{~min}$. The absorbance (a) value was detected by enzyme-linked immunosorbent assay at $490 \mathrm{~nm}$ wavelength and the results were recorded. U-118 cells were inoculated in 24 well culture plates. When the cells grew to about $80 \%$, serum free DMEM was replaced and cultured for $24 \mathrm{~h}$. When the cells grow to complete confluence, scratch the monolayer cells of each well with a $10 \mu \mathrm{l}$ sampling gun to create a scratch model of cultured cells. After scratch, rinse with Phosphate-buffered saline (PBS) twice. Mark at equal intervals along the edge of the scratch for inspection. After $24 \mathrm{~h}$ of culture, the wound healing degree was observed under the inverted microscope and photographed. The number of cells crossing the scratch boundary at $0 \mathrm{~h}$ was calculated and the percentage was compared with that of the control group. The Transwell chamber with $8 \mu \mathrm{m}$ microporous polycarbonate membrane was prepared and the matrix gel $(50 \mathrm{mg} / \mathrm{l})$ and serum free DMEM medium were diluted at the ratio of 1:3. $100 \mu$ l of diluted matrix glue was spread on the upper chamber of the chamber and sterile was kept overnight at $37 \%$. U-118 cells in logarithmic growth phase were collected. The cell concentration was adjusted to $2 \times 10^{8} / 1$ with DMEM medium. $200 \mu \mathrm{l}$ cell suspensions were added to the upper chamber in each well. DMEM medium was used to supplement to $1 \mathrm{ml}$. The supernatant of pre cultured U-118 cells was added into the lower chamber, $600 \mu \mathrm{l}$ per well. After incubation for $24 \mathrm{~h}$, the upper chamber was removed. The cells in the lower chamber were then stained with crystal violet and counted. The cell supernatant was discarded and Radio-Immunoprecipitation Assay (RIPA) cell lysate was added. The supernatant was centrifuged after $30 \mathrm{~min}$ of ice bath lysis. The protein solution $(30 \mu \mathrm{g})$ was added to $12 \%$ sodium dodecyl sulfate polyacrylamide gel electrophoresis (SDS-PAGE) gel for electrophoresis and $100 \mathrm{~V}$ electrophoresis was used to separate the protein completely. The membrane was transferred to PVDF membrane by wet methods and then transferred to PVDF membrane at $300 \mathrm{~mA}$ for $2 \mathrm{~h}$. The transferred film was sealed with $5 \%$ bovine serum albumin (BSA) sealing solution at $37 \%$ for 2 h. Phosphate Buffered Saline with Tween 20 (PBST) washed the membrane 3 times, 5 min each time, added 1:1000 diluted MMP-2 and MMP-9 primary antibody, and incubated overnight at 4\%. PBST was washed three times for $5 \mathrm{~min}$ each time. Horseradish Peroxidase (HRP) labeled secondary antibody was diluted with 1:5000 and incubated for $2 \mathrm{~h}$ at room temperature. PBST was washed three times for $5 \mathrm{~min}$ each time. Finally, Sensitive electrochemiluminescence (ECL) was used to develop and record. All data were analyzed by SPSS 20. The parameters of each group were expressed as mean \pm standard deviation $(x \pm s)$. After the homogeneity of F-test variance, t-test was used for comparison between groups. $\mathrm{p}<0.05$ was considered as significant difference. The U-118 cells were treated with different concentrations of plumbagin for $24 \mathrm{~h}$ and the morphological changes of U-118 cells were observed under inverted microscope. Under the microscope, the cells in the untreated group grew well, while the cells in the plumbagin group shrank in size, disappeared and separated from the surrounding cells in a dose dependent manner. MTT assay results showed that the proliferation of glioma cells decreased significantly in a concentration dependent manner $(0.5-1.0 \mathrm{mg} / 1$ group, $\mathrm{p}<0.05 ; 2.0-5.0 \mathrm{mg} / 1$ group, $\mathrm{p}<0.01$ ), indicating that a can significantly inhibit the proliferation of glioma cells. The half maximal inhibitory concentration $\left(\mathrm{IC}_{50}\right)$ of plumbagin on $\mathrm{U}-118$ cells was $14.7 \mu \mathrm{mol} / 1$, as shown in fig. 1 .

The results of scratch test showed that the U-118 cells in the control group migrated to the edge of the scratch rapidly after $24 \mathrm{~h}$ and the width of the scratch became narrower. After treatment with 10, 20, 50 and $100 \mu \mathrm{mol} / 1$ of plumbagin, the rate of cells crawling toward the scratch edge was significantly reduced and the repair of the scratch defect was slowed. In addition, 

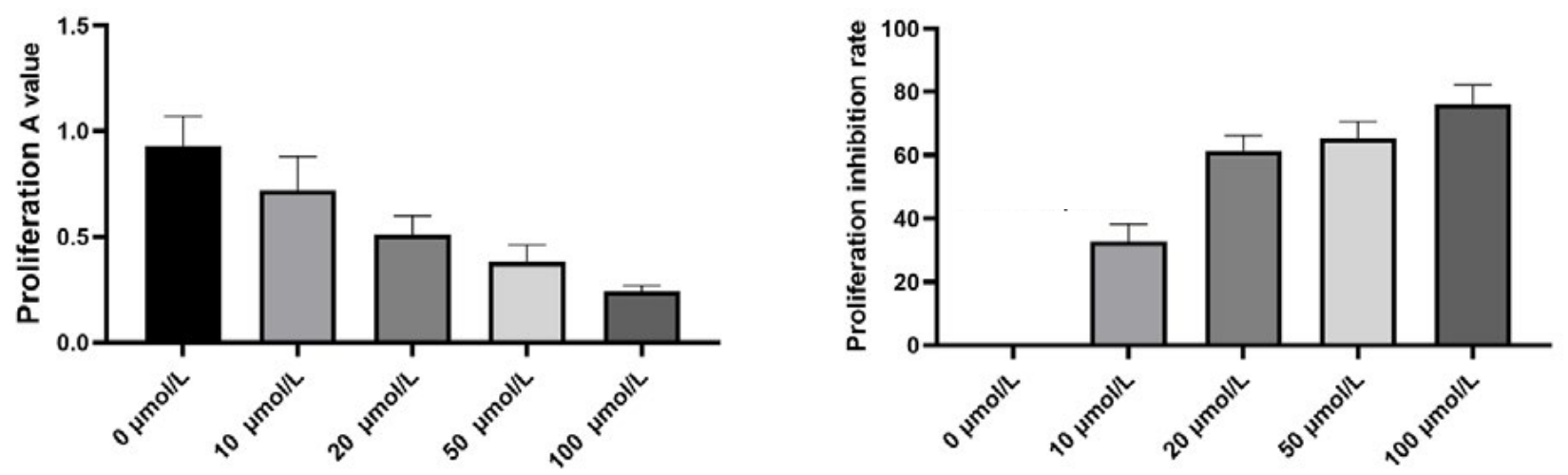

Fig. 1: Effects of different concentrations of plumbagin on the proliferation of U-118 cells

with the increase of the concentration of isoquinone, the plane movement ability of cells decreased significantly, as shown in fig. 2. Transwell analysis showed that the invasion of U-118 cells was significantly inhibited by plumbagin in a dose dependent manner, as shown in fig. 3. Compared with the control group, the expression of MMP-2 and MMP-9 protein in U-118 cell line was significantly decreased after $12 \mathrm{~h}$ treatment with different concentrations of plumbagin, while p21 protein expression was significantly increased with the increase of plumbagin concentration, as shown in fig. 4. Glioma is the most aggressive primary brain tumor, characterized by diffuse infiltration into normal brain parenchyma. Conventional standard treatment (surgical resection, adjuvant chemotherapy and radiotherapy) cannot completely cure glioma ${ }^{[8,9]}$. The prognosis of malignant glioma is poor and the median survival rate has not significantly improved in the past $40 y^{[10]}$. The antitumor effect of benzoquinone has been reported in the early studies ${ }^{[4]}$. It can inhibit the proliferation and induce apoptosis of glioma cells through p21, while there are few reports about the effect on cell migration and invasion. Our study showed that plumbagin significantly inhibited the growth of U-118 cells in a dose dependent manner. In addition, cell scratch and Transwell invasion results showed that plumbagin significantly decreased the migration and invasion of U-118 cells. Adhesion, as a starting condition for cancer cell invasion, cancer cells adhere to extracellular matrix components fibronectin (FN), LN and type IV collagen through integrins on the cell membrane, activate and secrete matrix metalloproteinases, degrade basement membrane and then migrate and move under the action of chemokines to achieve the results of invasion and metastasis ${ }^{[10,11]}$. The inhibition of adhesion, invasion and metastasis of cancer cells is the key to anti-tumor therapy and plumbagin inhibits the movement and invasion of U-118 cells. We speculate that even if some

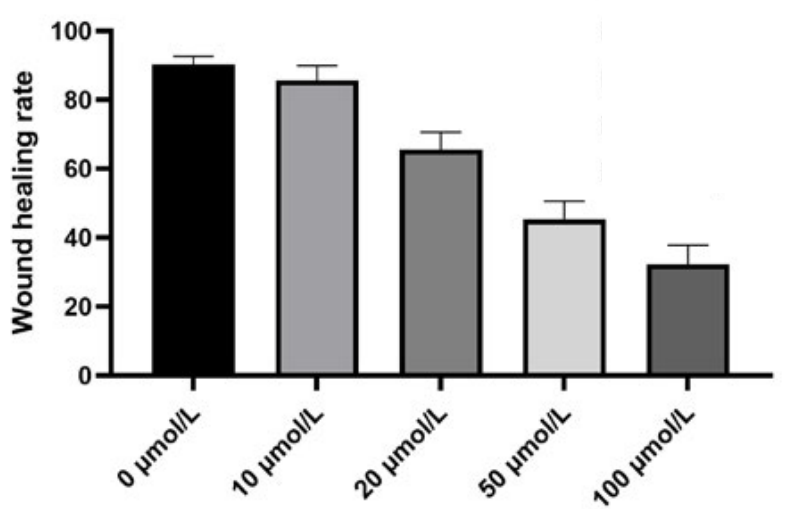

Fig. 2: Wound healing rate in scratch test

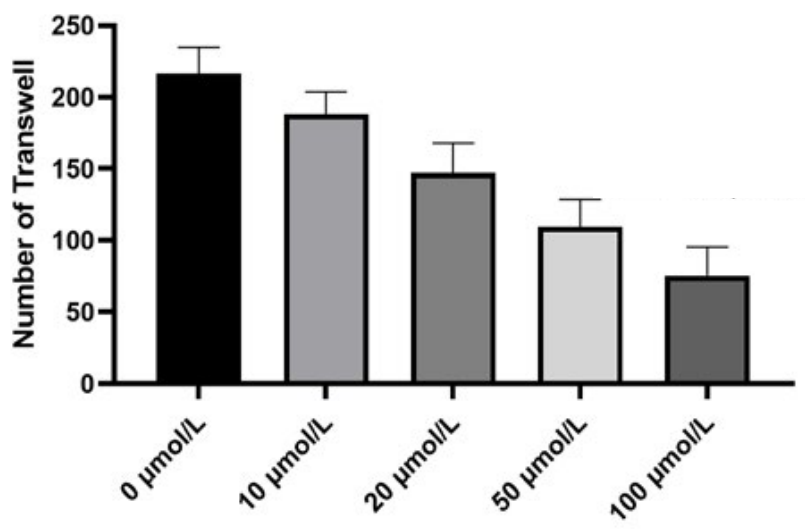

Fig. 3: The effect of plumbagin on invasion of U-118 cells

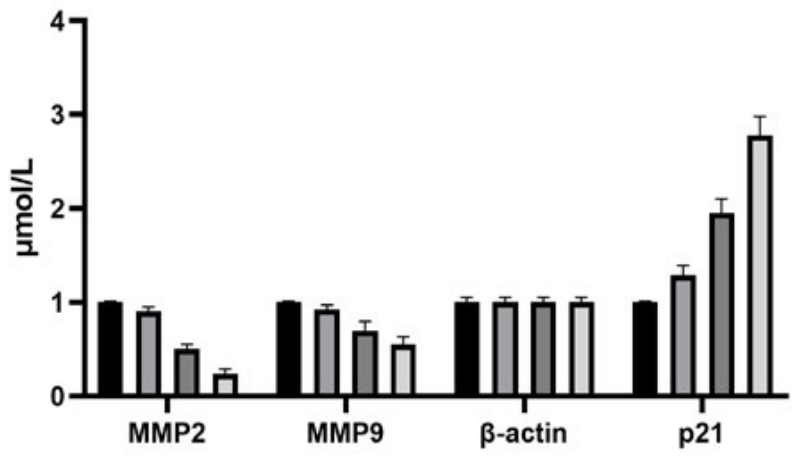

Fig. 4: Effects of plumbagin on the expression of p21, MMP-2 and MMP-9 in U-118 cells 
primary cancer cells are not killed, the probability of metastasis will be reduced due to the decrease of exercise ability, thus reducing the recurrence rate of glioma. The mechanism of plumbagin to U-118 cell motility and invasion has become a question of our thinking. In view of the fact that many molecules and signal pathways are involved in the process of tumor invasion and metastasis, these molecules and signal pathways play a role in all aspects of the tumor invasion and metastasis process and MMP is an important part of these molecules ${ }^{[12]}$. With the above resuIts, it is cIearly evident that plumbagin outdo many other existing drugs interms of patient recovery, quality of Iife and over aII satisfaction rate. Previous studies have reported that MMP is a key molecule in regulating the invasion and metastasis of glioma cells by various drugs and molecules and MMP-2 and MMP-9 are closely related to the prognosis of patients with glioma ${ }^{[13-16]}$. We further observed the changes of MMP-2 and MMP-9 in the process. We found that the protein expression of MMP2 and MMP-9 in U-118 cells decreased significantly after plumbagin treated U-118 cells, suggesting that plumbagin inhibits the invasion and metastasis of U-118 cells by inhibiting the expression of MMP-2 and MMP-9. In conclusion, plumbagin could inhibit the human glioma U-118 cell proliferation, invasion and migration. This effect may be achieved by inhibiting the expression of MMP-2 and MMP-9, but the specific mechanism needs further study.

\section{Conflicts of interest:}

The authors report no conflicts of interest.

\section{Acknowledgements:}

This work was supported by the National Natural Science Foundation of China (No. 81773803), the 2018 Research Startup Fund of Yanjing Medical College, Capital Medical University (No. 18qdky08).

\section{REFERENCES}

1. Lakka SS, Rao JS. Antiangiogenic therapy in brain tomors. Expert Rev Neurother 2008;8:1457-73.

2. Sathornsumetee $\mathrm{S}$, Rich JN. New approaches to primary brain tumor treatment. Anticancer Drug 2006;17:1003-16.
3. Wen PY, Kesari S. Malignant gliomas in adults. N Engl J Med 2008;359:492-507.

4. Ahmedin Jemal DVM, Rebecca Siegel, Ward E, Murray T, Xu J, Michael J. Cancer statistics. CA Cancer J Clin 2007;57:4366.

5. Azizi SA, Miyamoto C. Principles of treatment of malignant gliomas in adults: an overview. J Neurovirol 1998;4:204-16.

6. Colman H, Aldape K. Molecular predictors in glioblastoma: toward personalized therapy. Arch Neurol 2008;65:877-83.

7. Marosi C. Chemotherapy for malignant gliomas. Wien Med Wochenschr 2006;156:346-50.

8. Baruchel S, Diezi M, Hargrave D, Stempak D, Gammon $\mathrm{J}$, Moghrabi A, et al. Safety and pharmacokinetics of temozolomide using a dose-escalation, metronomic schedule in recurrent paediatric brain tumours. Eur J Cancer 2006;42:2335-42.

9. Kesari S, Schiff D, Doherty L, Gigas DC, Batchelor TT, Muzikansky A, et al. Phase II study of metronomic chemotherapy for recurrent malignant gliomas in adults. Neuro Oncol 2007;9:354-63.

10. Kim JT, Kim JS, Ko KW, Kong DS, Kang CM, Kim MH, et al. Metronomic treatment of temozolomide inhibits tumor cell growth through reduction of angiogenesis and augmentation of apoptosis in orthotopic models of gliomas. Oncol Rep 2006;16:33-9.

11. Aghi M, Cohen KS, Klein RJ, Scadden DT, Chiocca EA. Tumor stromal-derived factor-1 recruits vascular progenitors to mitotic neovasculature, where micro environment influences their differentiated phenotypes. Cancer Res 2006;66:9054-64.

12. Golden PL, Pollack GM. Blood-brain barrier efflux transport. J Pharm Sci 2003;92:1739-53.

13. Lee G, Schlichter L, Bendayan M, Bendayan R. Functional expression of P-glycoprotein in rat brain microglia. $\mathrm{J}$ Pharmacol Exp Ther 2001;299:204-12.

14. Miller DS, Bauer B, Hartz AM. Modulation of P-glycoprotein at the blood-brain barrier: opportunities to improve central nervous system pharmacotherapy. Pharmacol Rev 2008;60:196-209.

15. Jeliazkova-Mecheva VV, Bobilya DJ. A porcine astrocyte/ endothelial cell co-culture model of the BBB. Brain Res Protoc 2003;12:9-18.

16. Penas-Prado M, Gilber MR. Molecularly targeted therapies for malignant gliomas: advances and challenges. Expert Rev Anticancer Ther 2007;7:641-61.

This is an open access article distributed under the terms of the Creative Commons Attribution-NonCommercial-ShareAlike 3.0 License, which allows others to remix, tweak, and build upon the work non-commercially, as long as the author is credited and the new creations are licensed under the identical terms

This article was originally published in a special issue, "Clinical Research in Pharmaceutical and Biomedical Sciences" Indian J Pharm Sci 2021:83(1)Spl issue1;79-82 